\title{
The transient simulation of two-phase flow based on lattice Boltzmann method
}

\author{
Liang Chen ${ }^{1, a}$, Qin Yao, b and LuWu He ${ }^{1, \mathrm{c}}$ \\ ${ }^{1}$ School of Mechanical and Power Engineering, East China University of Science and Technology, \\ Shanghai 200237, China; \\ agodloveliang@163.com, byaoqing@mail.ecust.edu.cn, 'Luwuhe@ecust.edu.cn
}

Keywords: Two-phase flow, Transient simulation, False potential model, Lattice Boltzmann method.

\begin{abstract}
Shan-Doolen model which is based on Shan-Chen model, Combined of non-equilibrium extrapolation boundary and fully developed boundary treatment, and finally established Two-phase flow model. Applying this model, we firstly simulated the separation of two phase immiscible flow and represented the separation process of different time, so as to verify the feasibility of the model. Then the numerical simulation of transient flow of power law Newtonian two phase flow in the extended port was made to show the distribution density of different time. On this basis we clearly numerical analyse the whole process of change from the initial to the steady state so that we have a more clear understanding to the transient process of flow.
\end{abstract}

\section{1 introduction}

Multiphase flow is refers to the fluid system containing obvious boundary surface, such as liquid(gas) containing bubbles (droplet), immiscible liquids, and gas or liquid containing solid particles, etc. The fluid system in Nature and real engineering generally exist other substances more or less. There is almost no completely single-phase or one-component fluids. So strictly speaking the vast majority of flow problems belong to multiphase and multicomponent fluid flow problems. The macroscopic kinetic behavior of multiphase flow is very complex, often accompanied by some physical process such as diffusion, phase transition, interface components produce or sports and so on, and even many unknown chemical reaction happens. Because the many macroscopic behavior of multiphase flow is the embodiment of the components or interphase microscopic behavior, so using the traditional macro level of fluid dynamics simulation method to simulate the multiphase flow becomes very difficult. For example, in the macro level flow model of gas-liquid two phase flow we need to know all the state equation of each place, yet equation of near the interface is difficult to determine physically; For numerical simulation, the traditional method of CFD, such as: VOF, Level Set, etc., even can describe some of the more obvious interface changes, but it is difficult to capture or track more interface changes when they become smaller and more scattered. On the other hand, from the physical nature these macroscopic kinetic behavior of multiphase system are the result of the microscopic interaction between different components or different phase, also can saying is the macro representation. So, if we can put forward a kind of fluid model, making this kind of interaction that can be more accurately and clearly expressed, then we can express the complex flow problems from the bottom.

Because of the lattice Boltzmann method (LBM) itself has the characteristics of the microcosmic essence and the mesoscopic description, this is just for setting up multiphase flow model providing a feasible framework basis. For multiphase flow, there now is already a hot research topic in the field of LBM, having put forward many kinds of model till now, including ${ }^{]}$the color model proposed by Rothman [1], models according to the free energy theory [2,4] and models according to mesoscopic dynamics theory of multicomponent and multiphase flow in recent years such as HSD model and Guo model $[5,10]$. In order to accurately describe the given transient flow of power law Newtonian two phase flow, this article is based on the improved Shan-Chen model, and its correctness is proved by two phase separation model. Then the numerical simulation of power law - 
Newtonian transient flow of two phase flow in the extended port have been finished, so we can clearly numerical analyse the whole process of change from the initial to the steady state of.

\section{2 model and validation}

\subsection{Shan-Chen model}

For S different components of multicomponent fluid, Shan-Chen model also contains S distribution function evolution equation:

$$
f_{k i}\left(x+e_{i} \delta_{t}, t+\delta_{t}\right)-f_{k i}(x, t)=-\frac{1}{\tau_{k}}\left[f_{k i}-f_{k i}^{(e q)}\right], \mathrm{k}=1, \ldots, \mathrm{S}
$$

We assumes there is no the nonlocal interaction between fluid particles in Shan-Chen model:

$$
V_{k \bar{k}}\left(x, x^{\prime}\right)=G_{k \bar{k}}\left(\left|x-x^{\prime}\right|\right) \psi_{k}(x) \psi_{\bar{k}}\left(x^{\prime}\right)
$$

Where $G_{k \bar{k}}$ is related the Green function, which determines the strength of the interaction between the components. $\psi_{k}$ is related the effective density of component $k$. Different ways of expression, the equation of state is different. In general, function $G$ only need to consider the influence of adjacent lattice, for example in D2Q9:

$$
G_{k \bar{k}}\left(x, x^{\prime}\right)= \begin{cases}G_{k \bar{k}}, & \left|x-x^{\prime}\right|=\delta_{x} \\ 0, & \text { otherwise }\end{cases}
$$

Where the absolute value of $G_{k \bar{k}}$ decide the strength of the interaction between the components $k$ and $\bar{k}$, And its symbol determines the nature of the interaction force, that is to say the negative represent attractive force and positive represent the repelling force. According to the interaction potential, we can get interaction force between particles of composition $k$ particles:

$$
F_{k}(\mathbf{x})=-\psi_{k}(\mathbf{x}) \sum_{\bar{k}} G\left(\mathbf{x}, \mathbf{x}^{\prime}\right) \sum_{i} \psi_{\bar{k}}\left(\mathbf{x}^{\prime}\right) \mathbf{e}_{i}
$$

Shan-Chen model reflect the influence of the interaction force between the particles By equilibrium speed:

$$
u_{k}^{e q}(x)=u(x)+\tau_{k} \delta_{t} \frac{F_{k}}{\rho_{k}}
$$

where $u$ is the speed of the mixed fluid:

$$
\rho u=\sum_{k} \rho_{k} u_{k}=\sum_{k} \sum_{i} c_{i} f_{k i}
$$

$\rho=\sum_{k} \rho_{k}$ is density of mixed fluid.

After the Shan-Chen model had been presented, Shan and Doolen aimed at some problems improved the basic model again [11]. First, the improved model have a new definition to equilibrium speed of the equilibrium distribution function:

$$
u_{k}^{e q}=u^{\prime}+\tau_{k} \delta_{t} \frac{F}{\rho_{k}}
$$

Where $u^{\prime}$ should make sure that collision process can conform to the law of conservation of momentum in the absence of force, that is:

$$
u^{\prime}=\sum_{k} \frac{\rho_{k} u_{k}}{\tau_{k}} / \sum_{k} \frac{\rho_{k}}{\tau_{k}}
$$

In the Shan and Doolen model, speed of mixed fluid is:

$$
u(x)=\left(\sum_{k} \rho_{k} u_{k}+\frac{\delta_{t}}{2} \sum_{k} F_{k}\right) / \rho
$$

Macroscopic fluid variables s expressed as below: 
Pressure :

$$
\begin{gathered}
p(x)=c_{s}^{2} \sum_{k} \rho_{k}(x)+\frac{3}{2} \sum_{\bar{k}} G \psi_{k}(x) \psi_{\bar{k}}(x) \\
v=\left(\sum_{k} x_{k} \tau_{k}-\frac{1}{2}\right) / c_{s}^{2}
\end{gathered}
$$

\subsection{Simulation of mix of two phase separation process of lattice Boltzmann method}

As is shown in the picture, the simulate area is $200 \times 200$, that is to say $L_{x}=L_{y}=200$, The initial viscosity omega $1=$ omega $2=1$, parameter $G_{12}=1.2$.

The initial conditions: Equilibrium speed: $u_{k}^{e q}=0,(\mathrm{k}=1,2)$; Density: $\rho_{1}(\mathrm{x}, \mathrm{y})$ is to any value between 0 and $1 ; \rho_{2}=1.0$.

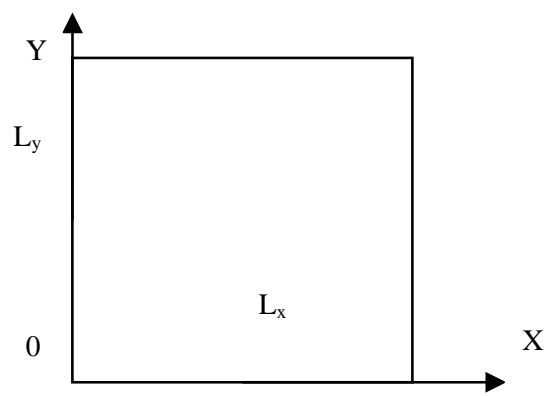

Fig2.1 The model of two phases of unmixing

Boundary processing: All boundary using nonequilibrium extrapolation method.

Simulation results are shown in figure 2.2, from the results we can concluded that the immiscible two-phase flow have a good separation, the results are consistent with existing analysis result, and

verify the feasibility of the model at the same time.
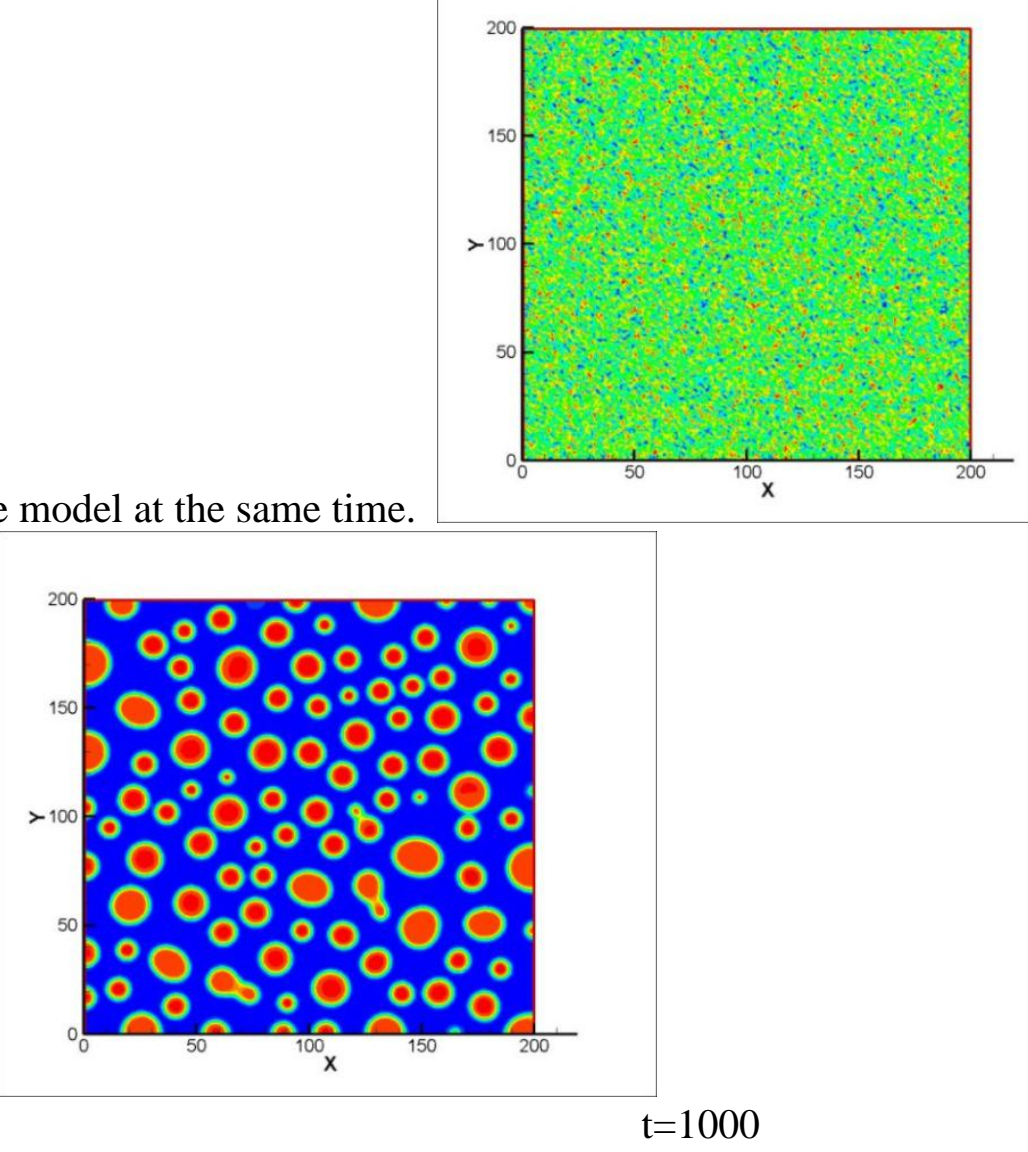


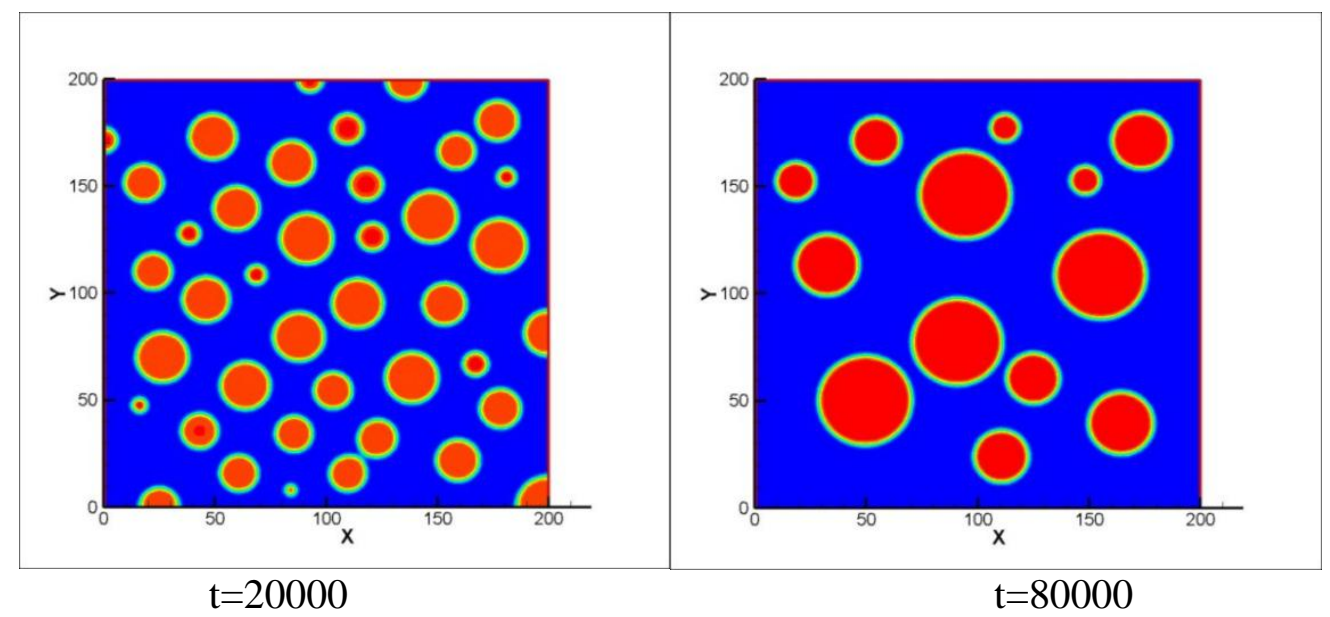

Fig2.2 Separation process in different times

\section{3 transient simulation of two phase fluid in an extension flow of Lattice Boltzmann method}

Geometry graph of extension flow is shown in figure 3.1, all boundaries except the inward and outward orifice are fixed wall, and computing area is $90 \times 180$.Inlet level $h=30$, outlet level $H=90$, The entrance length $L_{1}=60$, The total length $L=3 L_{1}=180$,Inlet velocity $u_{0}$ is constant.

The initial conditions: as shown in figure 3.1, The left side of the dotted line is phase 1 for power law fluid, $\rho_{1}(\mathrm{x}, \mathrm{y})=2, \rho_{2}(\mathrm{x}, \mathrm{y})=0$. the right is the phase 2 for Newtonian fluid, $\rho_{1}(\mathrm{x}, \mathrm{y})=0, \rho_{2}$

$(\mathrm{x}, \mathrm{y})=1$. omega1 $=1.38$,omega $2=1, G_{12}=1.2, R e=1$. Inlet equilibrium rate: $u_{k}^{e q}=\mathrm{u}_{0}(\mathrm{k}=1,2)$, Equilibrium rate of other areas: $u_{k}^{e q}=0 \quad(\mathrm{k}=1,2)$,Power law index $\mathrm{n}=0.9$.

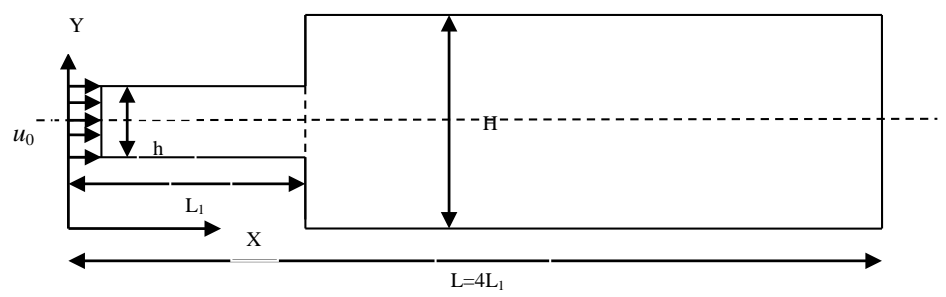

Fig.3.1 The 2D planar viscoelastic sudden expansion flow

Boundary processing: right boundary use full development processing format, the rest place use non-equilibrium extrapolation scheme.

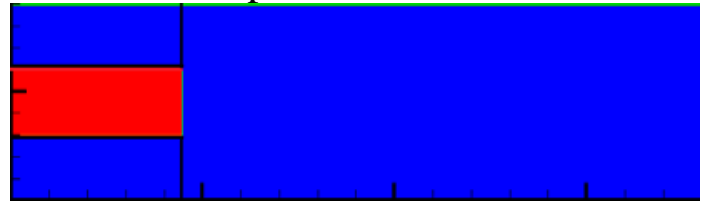

$\mathrm{t}=0$

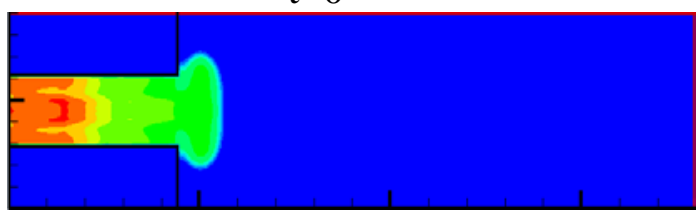

$t=100$

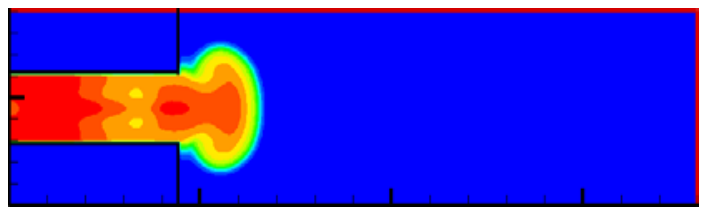

$t=200$

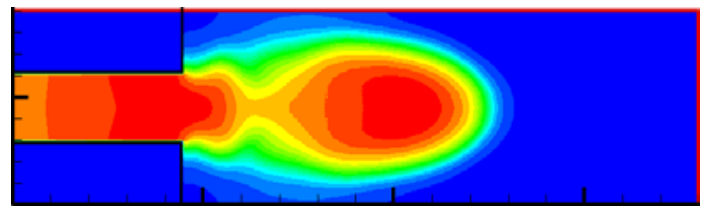

$\mathrm{t}=1000$

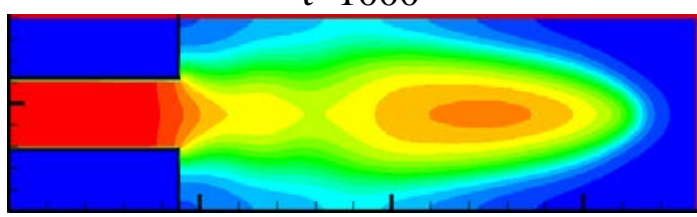

$t=2000$

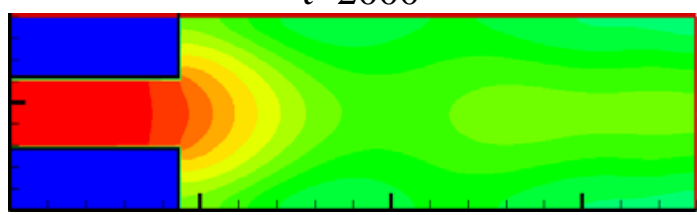

$t=10000$ 


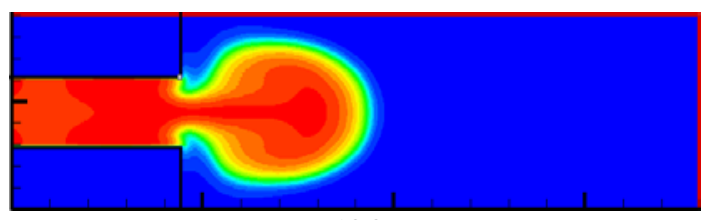

$\mathrm{t}=500$

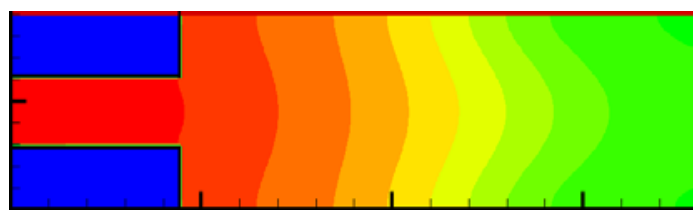

$\mathrm{t}=40000$

Fig3.2 Density distribution in different times for power-law flow and Newtonian flow

When $n=0.9$,figure 3.2 lists the density distribution state in the process of expansion flow of power law - Newton two phase fluid in different time, shows the change of the two-phase fluid in different time from just contact to the steady state, depicts the transient change process clearly.

\section{4 conclusion}

This paper introduces Shan-Chen model of multiphase flow in detail, and using the improved Shan - Doolen model, we established the two phase flow model by Combination of non-equilibrium extrapolation boundary processing format and full development of border processing format. Applying this model, firstly we simulated the separation of immiscible two phase flow, and represented the separation process of different time, so as to verify the feasibility of the model. Then we simulated the extension model of the power law - Newton two phase fluid by also using the full development of border processing format and non-equilibrium extrapolation boundary processing format, showing the density distribution of different time. On this basis we have a more clear understanding for the transient flow process. Compared with static research, this paper focuses on introducing the whole process of change from the initial point to the steady state, that is a transient study, which have more practical significance.

\section{References}

[1] Rothman D H, Keller J M. Immiscible cellular-automaton fluids[J]. Journal of Statistical Physics, 1988, 52(3):1119-1127.

[2] Orlandini E, Swift M R, Yeomans J M. A lattice Boltzmann model of Binary-fluid mixtures[J]. Europhysics Letters, 2007, 32(6):463.

[3] Swift M R, Osborn W R, Yeomans J M. Lattice Boltzmann simulation of nonideal fluids[J]. Physical Review Letters, 1 995,75(5):830-833.

[4] Swift M R,Orlandini E, Osborn W R, et a1. Lattice Boltzmann simulations of liquid-gas and binary fluid systems[J]. Physical Review E, 1996, 54(5):504I-5052.

[5] Shan Xiaowen. Multicomponent lattice Boltzmann model from continuum kinetic theory[J]. Physical Review E, 2010, 81(4): 045701.1.045701.4.

[6] Luo L S. Unified theory of lattice Boltzmann models for nonideal gases[J]. Physical Review Letters, 1998, 8l(8):1618-1621.

[7] He X, Shan X, Doolen D. Discrete Boltzmann equation model for nonideal gases[J]. Physical Review E, 1998, 57(1):13-16.

[8] He X, Chen S, Zhang R. A lattice Boltzmann scheme for incompressible multiphase flow and its application in simulation of Rayleigh-Taylor instability[J]. Journal of Computational Physics, 1999, 152(2):642-663.

[9] Guo Z. Zhao T S. Discrete velocity and lattice Boltzmann models for binary mixtures of nonideal fluids[J]. Physical Review E, 2003, 68(3):035302-1-035302-7.

[10]Guo Z, Zhao T S. Finite-difference-based lattice Boltzmann model for dense binary mixtures[J]. Physical Review E, 2005, 71(2):026701-1.026701-6.

[11]D. H. Rothman and J.M.Keller. Immiscible cellular-automation fluids [J]. Comput. Phys, 2002, 
179, 439-451. 